\title{
La asistencia privada en el Sistema Nacional de Salud
}

\author{
Private assistance in the National Health System
}

Jaime Lozano-Alcázar*

Académico emérito, expresidente de la Academia Mexicana de Cirugía, director médico de la Fundación Hospital Nuestra Señora de la Luz, Ciudad de México, México

A menudo en las conferencias, las sesiones y las estadísticas generales de la salud en México se olvidan de incorporar las contribuciones de las instituciones de asistencia privada.

En la época virreinal, la Iglesia Católica fue la entidad por antonomasia dedicada a las labores sociales. Las órdenes religiosas, al extender la labor evangelizadora, iban fundando escuelas, hospicios, asilos y hospitales. Sin embargo, desde el mismo siglo xvi se inició en la Nueva España la instalación de fundaciones asistenciales independientes de la voluntad de los jerarcas del clero, y por lo tanto no vinculadas estrictamente a la Iglesia, aunque habitualmente eran servidas por religiosos de las órdenes monásticas que se asentaban en el territorio conquistado.

La que se define como primera institución de asistencia privada fundada en el país se debe a la voluntad del conquistador Hernán Cortés, que dispuso una cantidad para su fundación, la cual se llevó a cabo en 1524, apenas 3 años después de la caída de Tenochtitlán ${ }^{1}$. Se trata del Hospital de la Inmaculada Concepción de Nuestra Señora y de Jesús Nazareno, que subsiste hasta hoy y se conoce como Hospital de Jesús, que conserva su ubicación original en el número 82 de la actual avenida 20 de Noviembre, en el centro de la Ciudad de México; según una versión de la tradición, en el sitio del primer encuentro entre el extremeño y Moctezuma II. En la voluntad testamentaria se enfatizaba que estaría dedicado a los heridos durante la guerra de conquista y a la atención de los hijos de los caídos en defensa de la capital azteca ${ }^{2}$.
El Dr. Pedro López, licenciado en Medicina por la Universidad de Valladolid (Castilla, España), título luego convalidado por la Universidad de México, la que le otorgó más tarde el doctorado, después de duros esfuerzos logró tener medios para fundar el Hospital de san Lázaro en 1572, dedicado a pacientes afectados de lepra, el primero en su clase en el continente, y en 1582 el Hospital de los Desamparados ${ }^{3}$.

El Dr. López tenía amistad con Fray Bernardino Álvarez, otro entusiasta creador de hospitales. Fundó la Orden de San Hipólito de la Caridad, más conocida como Los Hipólitos, dedicados a la atención de los pacientes dementes. En el convento anexo al templo de San Hipólito, que hizo construir, en la actual avenida Puente de Alvarado en la Ciudad de México, fray Bernardino instaló en 1566 el primer hospital de América dedicado a los alienados. En 1586 fundó en Oaxtepec, en el actual Estado de Morelos, el Hospital de Santa Cruz, también para enfermos mentales, y no se detuvo ahí, sino que continuó su benemérita labor fundando hospitales en Acapulco, Puebla, Xalapa e incluso en La Habana $^{3,4}$. Fray Bernardino también ejercía la medicina.

A lo largo del período virreinal, especialmente en los siglos xvi y xvii, se continuaron fundando otros hospitales laicos gracias a las aportaciones de corporaciones, como el Hospital de San Juan de Letrán en Puebla, el de Nuestra Señora de los Remedios en Campeche, el de Nuestra Señora del Rosario en Mérida, el de la Caridad del Nombre de Dios en Durango,

\footnotetext{
Correspondencia:

*Jaime Lozano-Alcázar

Pestalozzi 1204-803

Col. del Valle, Del. Benito Juárez

C.P. 03100, Ciudad de México, México

E-mail: drjaimelozano@gmail.com
}

Fecha de recepción: 17-05-2018

Fecha de aceptación: 30-05-2018

DOI: 10.24875/CIRU.M18000059
Cir Cir. 2018;86:385-387

Contents available at PubMed www.cirugiaycirujanos.com 
el de la Santa Veracruz en Valladolid (Yucatán), el de Nuestra Señora de Montserrat en la Ciudad de México y el de San Cosme y San Damián en Veracruz, por mencionar solo algunos ${ }^{3}$. El último nosocomio creado durante la época virreinal fue el de San Sebastián, en 1799, en la ciudad de Veracruz ${ }^{5}$.

Con el devenir del tiempo esos hospitales tuvieron disímbolos destinos: algunos desaparecieron, otros conservan nombre y vocación, y otros recibieron un nuevo nombre laico a partir de las reformas liberales. Un conocido ejemplo es el actual Hospital Juárez, nacido como Hospital de San Pablo, pues el antiguo Colegio de San Pablo de los agustinos fue convertido en hospital ante las necesidades impuestas por la guerra contra los EE.UU. en el año de 1847.

Como vemos, a aquella primera institución de asistencia privada, el Hospital de Jesús, se fueron agregando otras instituciones filantrópicas preocupadas en especial por la población en condiciones de vulnerabilidad; de hecho, don Valentín Gómez Farías, en 1833, siendo presidente de la República, trató de crear un sistema de asistencia que se encargara de la población más pobre'.

La Ley de Desamortización de las Fincas Rústicas y Urbanas de las Corporaciones Civiles y Religiosas de México, conocida como Ley Lerdo y promulgada en 1856 por Ignacio Comonfort como presidente de la República, así como el conjunto de ordenamientos llamado Leyes de Reforma, llevaron a que la Iglesia dejara de manejar numerosas instituciones asistenciales, quedando la mayor responsabilidad de asistencia pública en manos del Estado. El gobierno de la República no se desentendió de las necesidades de la población; el presidente Benito Juárez, en 1861, creó la Dirección General de Fondos de Beneficencia, que, exentos de impuestos, podían invertirse exclusivamente en obras de beneficencia. La Beneficencia Pública quedó adscrita al gobierno del Distrito Federal'.

Para mejorar el control y las funciones de las instituciones de beneficencia, y evitar situaciones fraudulentas, el general Porfirio Díaz, el 7 de noviembre de 1899, promulgó la Ley de Instituciones de Asistencia Privada para el Distrito Federal con el fin de auspiciar, promover y proteger las iniciativas de los particulares a favor de los más necesitados. En esa ley se establece la creación de la Junta de Asistencia Privada (JAP), que vigila la buena marcha de las instituciones de asistencia privada en la Ciudad de México. Las diferentes instituciones cobijadas en la JAP se agrupan en seis rubros: 1) Educación, cultura y desarrollo comunitario; 2) Niñas, niños y adolescentes; 3) Personas mayores; 4) Discapacidad y rehabilitación; 5) Salud y adicciones; y 6) Donantes y prendarias. En el rubro de Salud y adicciones se integran 81 instituciones, entre las que se cuentan la Beneficencia Española, el Hospital Escandón, la Cruz Roja Mexicana, el Hospital de Jesús y The American British Cowdray Medical Center, por mencionar algunos'.

En ese rubro de instituciones de asistencia privada dedicadas a salud y las adicciones se encuentran tres hospitales de atención a enfermos de los ojos, a las que me voy a referir en particular por ser mi especialidad la oftalmología y porque conozco mejor sus alcances y funcionamiento. En orden de antigüedad, la Fundación Hospital Nuestra Señora de la Luz I.A.P., originada en 1876 por el legado testamentario de don Ignacio Valdivielso y Vidal de Lorca, quinto conde de San Pedro del Álamo; la Asociación para Evitar la Ceguera en México, I. A. P., fundada en 1918 por un grupo de filántropos encabezados por el Dr. José Terrés; y la Fundación Conde de Valenciana, Instituto de Oftalmología, en 1976, gracias a la herencia dejada por don Luis Ludert y Rull, conde de la Valenciana. Las tres instituciones gentilmente proporcionaron los datos que a continuación señalo.

Tan solo en el año pasado, 2017, en conjunto los médicos de estas tres instituciones dedicadas a la oftalmología llevaron a cabo, en números redondos, 440,000 consultas de la especialidad, 31,000 cirugías oftalmológicas y 60,000 tratamientos a los ojos (procedimientos con láser, inyecciones intravítreas, etc.), además de 60,000 consultas de otras especialidades (cardiología, medicina interna, inmunología, etc.) en sus servicios auxiliares. Se calcula que estos tres hospitales proporcionan el $50 \%$ de la atención oftalmológica a los mexicanos.

Creo que las cifras mencionadas son elocuentes por sí mismas en cuanto a la importancia de estas tres instituciones para la salud visual del país; además, si a estas cifras sumamos los cientos de miles más de pacientes que se atienden en las diferentes instituciones de salud de la asistencia privada, se pone de manifiesto la contribución muy importante que dan al Sistema Nacional de Salud, lo que, como decía, me parece que pocas veces es reconocido en las estadísticas oficiales. A mayor abundamiento, la mayoría de las instituciones de asistencia privada conservan la vocación altruista que les dio origen y atienden a 
pacientes en situación económica vulnerable, o bien tienen un programa de atención asistencial como parte de sus actividades; desde luego, la labor social que desarrollan es muy trascendente. Podemos imaginar el caos que ocasionaría a nuestros sistemas de seguridad y de protección social, ya saturados, que todos estos pacientes demandaran sus servicios, con mayor razón si consideramos que, según la página electrónica del 29 de mayo de 2018 de la revista Forbes, en México, según datos del Instituto Nacional de Estadística y Geografía (INEGI), de los 112.33 millones de mexicanos solo el $10 \%$ tiene acceso al $41.7 \%$ de los recursos que genera el país anualmente ${ }^{6}$, es decir, solo una proporción menor de los mexicanos puede pagar la atención de la medicina privada, y el resto debe recurrir a los sistemas de seguridad social del Estado o a las instituciones de asistencia privada. La medicina privada y las instituciones de salud de asistencia privada son soportes indispensables de nuestro Sistema Nacional de Salud.

\section{Referencias}

1. Junta de Asistencia Privada del Distrito Federal (Consultado el 29/05/18) Disponible en: http://jap.org.mx/portal/index.php?lang=es

2. Hospital de Jesús Nazareno. Wikipedia (Consultado el 29/05/18) Disponible en: https://en.wikipedia.org/wiki/Hospital_de_Jesús_Nazareno

3. Rodríguez-Sala ML, Ramírez V, Tolentino A, Rivera C, Pérez A, Mireles A. Los cirujanos de hospitales de la Nueva España (siglos XVI y XVII): ¿miembros de un estamento profesional o de una comunidad científica? Universidad Nacional Autónoma de México, Instituto de Investigaciones Sociales, Academia Mexicana de Cirugía, Patronato del Hospital de Jesús, Secretaría de Salubridad y Asistencia. 1..$^{\text {a }}$ ed. 2005.

4. Bernardino Álvarez. Wikipedia (Consultado el 29/05/18) Disponible en: https://commons.wikimedia.org/wiki/Category:Bernardino_Álvarez.

5. Loyo-Varela M, Díaz-Cházaro H. Hospitales en México. Cir Cir. 2009;77:497-504.

6. Baltasar Madrid Nieto. El papel de las grandes empresas en la asistencia privada. Forbes México (Consultado el 29/05/18) Disponible en: https://www.forbes.com.mx/el-papel-de-las-grandes-empresas-en-laasistencia-privada/ 